\title{
A Bayesian inference approach to unveil supply curves in electricity markets
}

\author{
Mitridati, Lesia Marie-Jeanne Mariane; Pinson, Pierre
}

Published in:

IEEE Transactions on Power Systems

Link to article, DOI:

10.1109/TPWRS.2017.2757980

Publication date:

2017

Document Version

Peer reviewed version

Link back to DTU Orbit

Citation (APA):

Mitridati, L. M-J. M., \& Pinson, P. (2017). A Bayesian inference approach to unveil supply curves in electricity markets. IEEE Transactions on Power Systems, 33(3), 2610-2620.

https://doi.org/10.1109/TPWRS.2017.2757980

\section{General rights}

Copyright and moral rights for the publications made accessible in the public portal are retained by the authors and/or other copyright owners and it is a condition of accessing publications that users recognise and abide by the legal requirements associated with these rights.

- Users may download and print one copy of any publication from the public portal for the purpose of private study or research.

- You may not further distribute the material or use it for any profit-making activity or commercial gain

- You may freely distribute the URL identifying the publication in the public portal

If you believe that this document breaches copyright please contact us providing details, and we will remove access to the work immediately and investigate your claim. 


\title{
A Bayesian Inference Approach to Unveil Supply Curves in Electricity Markets
}

\author{
Lesia Mitridati, Pierre Pinson, Senior Member, IEEE
}

\begin{abstract}
With increased competition in wholesale electricity markets, the need for new decision-making tools for strategic producers has arisen. Optimal bidding strategies have traditionally been modeled as stochastic profit maximization problems. However, for producers with non-negligible market power, modeling the interactions with rival participants is fundamental. This can be achieved through equilibrium and hierarchical optimization models. The efficiency of these methods relies on the strategic producer's ability to model rival participants' behavior and supply curve. But a substantial gap remains in the literature on modeling this uncertainty. In this study we introduce a Bayesian inference approach to reveal the aggregate supply curve in a day-ahead electricity market. The proposed algorithm relies on Markov Chain Monte Carlo and Sequential Monte Carlo methods. The major appeal of this approach is that it provides a complete model of the uncertainty of the aggregate supply curve, through an estimate of its posterior distribution. We show on a small case study that we are able to reveal accurately the aggregate supply curve with no prior information on rival participants. Finally we show how this piece of information can be used by a price-maker producer in order to devise an optimal bidding strategy.
\end{abstract}

Index Terms-Bayesian inference, Sequential Monte Carlo, Markov Chain Monte Carlo, Strategic bidding

\section{NOMENCLATURE}

\section{Indexes}

$d \quad$ Index for days $(d=1, \ldots, D)$

$h \quad$ Index for hours $(h=1, \ldots, H)$

$w \quad$ Index for wind producers $(w=1, \ldots, W)$

$g \quad$ Index for market participants $(g=1, \ldots, G)$

$b \quad$ Index for generation blocks $(b=1, \ldots, B)$

$m \quad$ Index for Markov Chain Monte Carlo iterations $(m=1, \ldots, M)$

$i$ Index for Sequential Monte Carlo particles $(i=$ $1, \ldots, N)$

\section{Market Clearing Variables and Parameters}

$$
\begin{array}{ll}
\lambda_{d, h}^{\text {spot }} & \text { Spot price at hour } h \text { of day } d, \text { in } € / \mathrm{MWh} \\
P_{g, d, h} & \begin{array}{l}
\text { Power output of market participant } g \text { at hour } h \text { of } \\
\text { day } d, \text { in MWh }
\end{array} \\
P_{w, d, h} & \begin{array}{l}
\text { Power output of wind producer } w \text { at hour } h \text { of } \\
\text { day } d, \text { in MWh }
\end{array}
\end{array}
$$

L. Mitridati and P. Pinson are with the Technical University of Denmark, Department of Electrical Engineering, Centre for Electric Power and Energy, Kgs. Lyngby, Denmark (email: \{lemitri,ppin\}@dtu.dk)

The authors are partly supported by the Danish Innovation Fund through the projects 5s - Future Electricity Markets (12-132636/DSF) and CITIES (DSF1305-00027B), as well as EUDP through the project EnergyLab Nordhavn (EUDP 64015-0055).

\begin{abstract}
$\bar{P}_{g, d, h} \quad$ Offered quantity of market participant $g$ at hour $h$ of day $d$, in MWh

$\bar{P}_{w, d, h} \quad$ Wind power available from producer $w$ at hour $h$ of day $d$, in MWh

$L_{d, h} \quad$ Electricity load at hour $h$ of day $d$, in MWh

$S F_{g, d, h} \quad$ Supply function of market participant $g$ at hour $h$ of day $d$, in $€ / \mathrm{MWh}$

$\alpha_{g, d, h} \quad$ Supply function parameter of market participant $g$ at hour $h$ of day $d$, in $€ / \mathrm{MWh}^{2}$

$\beta_{g, d, h} \quad$ Supply function parameter of market participant $g$ at hour $h$ of day $d$, in $€ / M W h$

$\bar{R}_{g} \quad$ Ramp-up limit of market participant $g$, in MWh

$\bar{P}_{g} \quad$ Maximum power output of market participant $g$, in $\mathrm{MWh}$
\end{abstract}

\section{Hidden Markov Model States and Parameters}

$\Lambda_{b, d} \quad$ Random vector of latent states of generation block $b$ in day $d$

$\Lambda_{d} \quad$ Random vector of latent states of all generation blocks in day $d$

$Y_{d} \quad$ Random vector of observable states in day $d$

$\Theta \quad$ Random vector of static model parameters

$\mu_{b, \theta}($.$) \quad Initial density of the latent states \Lambda_{b, 1: D}$

$f_{b, \theta}($.$) \quad Transition function of the latent states \Lambda_{b, 1: D}$

$\mu_{\theta}($.$) \quad Initial density of the latent states \Lambda_{1: D}$

$f_{\theta}($.$) \quad Transition function of the latent states \Lambda_{1: D}$

$\mathcal{L}_{\theta}($.$) \quad Observations likelihood$

$\pi($.$) \quad Prior density of the static parameters \Theta$

\section{Markov Chain Monte Carlo Algorithm Notations}

$M_{0} \quad$ Number of burn-in iterations

$q($.$) \quad Proposal density$

$\alpha($.$) \quad Acceptance probability$

$K($.$) \quad Markov chain transition kernel$

\section{Sequential Monte Carlo Algorithm Notations}

$q($.$) \quad Importance function$

$w_{d}^{(i)} \quad$ Normalized importance weight of the particle $\lambda_{d}^{(i)}$

$A_{d}^{i} \quad$ Ancestral index of the particle $\lambda_{d}^{(i)}$

$\hat{p}($.$) \quad Monte Carlo approximation of target density p($.

\section{INTRODUCTION}

$\mathbf{T}$ HE liberalization of the power sector has led to the development of wholesale electricity markets. However, high levels of market concentration and oligopolistic conditions still prevail in various countries [1]. Additionally, due to 
the technical characteristics of the electricity supply industry, market power is only imperfectly correlated with concentration measures and can be exercised by small firms during peak hours [2], [3]. Ref. [4] and [5] present an overview of market power issues in various countries, and market designs to mitigate them. In particular, over the last decade regulators have focused on monitoring competition behaviors, and increasing market data transparency [6], [7].

In electricity markets, producers with non-negligible market power bid a supply function that deviates form their true marginal cost in order to can increase their profit [8], [9]. The issue of optimal bidding for strategic producers has traditionally been addressed in the literature as a profit maximization problem under uncertainty. The authors in [10] modeled the optimal bidding strategy of a producer on a wholesale market as a robust optimization problem, introducing uncertainty in market prices through confidence intervals. And, in [11] the strategic offering of a wind power producer, for different risk preferences, is formulated as a two-stage stochastic optimization problem. Uncertainty in wind production, day-ahead and real-time prices is introduced through a finite number of scenarios. However, for participants with non-negligible market power, modeling the uncertainty of rival participants' behavior is essential. Novel approaches relying on game theory, agent-based models [12], [13], equilibrium and hierarchical optimization [14] enable us to design decision-making tools modeling the interactions between rival participants.

A recently published work introduced a stochastic multilayer agent-based model of electricity markets, accounting for uncertainty of rival participants' behavior [15]. Ref. [16] used evolutionary games and near Nash equilibrium to model competition in electricity markets and predict local marginal prices. Additionally, the authors in [17] implemented the agent-based reasoning through a Dynamic Bayesian Network (DBN) representation and proposed an online Bayesian learning algorithm to predict loads and Residual Demand Curves (RDC). As discussed in [18], RDCs are commonly employed to model and predict the behavior of rival participants. Based on observed or predicted aggregate demand and supply curves, each agent builds its individual RDC and optimal bidding strategy. Finally, the authors in [19]-[22] formulated the optimal bidding strategy of a price-maker wind producer in the day-ahead and balancing markets as a stochastic hierarchical optimization problem. In this model the market clearing is explicitly formulated as a constraint of the producer's profit maximization problem. This approach showed a significant improvement in bidding performances, provided that an accurate description of the aggregate supply curve in the electricity market is available. Similarly, complementarity theory can be used to model the optimal dispatch of combined heat and power plants in sequential heat and electricity markets [23]. This literature provides useful insight on the strategic behavior of market participants in oligopolistic markets. The efficiency of these methods relies on an accurate modeling of uncertainty sources. The issue of wind production, spot prices and load forecasting has been extensively addressed in the literature [24]. However, a gap remains in the description of the probability density of the aggregate supply curve.
The aforementioned studies suggest that a model for predicting the aggregate supply curve in an electricity market is a key piece of information for strategic producers to make informed decisions. Ref. [25] introduced the bid function equilibrium model, predicting supply curves of participants in an electricity market based on available data on their marginal costs. The predictions of this model were validated in a study comparing the theoretical results to historical bids submitted to the British spot market [26]. Additionally, the authors in [27] reviewed two methods for aggregate supply curves prediction based on principal component analysis and reduced rank regression, using data on past supply curves. Finally, as discussed in [28] the RealScen model is an integrated tool that collects and analysis historic market participants' bids data, and generates realistic bids scenarios using artificial neural networks, support vector models and simple average methods. Combined with agent-based [12] or complementarity models of electricity markets, the RealScen provides a powerful decision-making tool for strategic producers.

However, information on rival participants' historic bids or marginal costs is seldom available due to market operators' confidentiality policies. This type of information is valuable for both electricity producers to design optimal bidding strategies, and market regulators to monitor competition behaviors [4]-[7]. To the best of our knowledge, only one paper proposed a method for inferring these bids based on available market data [29]. Rival participants' offering bids on an electricity market are revealed using an inverse optimization problem. This approach provides an accurate estimation of the marginal cost of each participant, provided the dispatched production of each generation block and all the generators' technical characteristics are perfectly known. In practice these assumptions are quite restrictive. Additionally, this approach does not provide a model of the uncertainty of the offering bids.

That is why we propose a more flexible method, allowing us to reveal the aggregate supply curve in an electricity market solely based on historic market data. Bayesian inference enables us to update our prior belief on the unknown states of the system, e.g. supply curves, as new information is acquired through the observed states. Since exact inference methods, such as the exact expectation-maximization algorithm, are often computationally intractable (see [30] and [31]), we focus on approximate stochastic inference methods, such as Markov Chain Monte Carlo (MCMC) [32]-[35] and Sequential Monte Carlo (SMC) [36]-[40] algorithms. The aim of stochastic inference algorithms is to iteratively build a large sample, approximately distributed according to the distribution of interest. These methods provide a description of the uncertainty of the unknown states, through an estimate of their posterior distribution, from which it is easy to sample. That is why they have found applications in various research fields, such as finance [41], multi-target tracking [42] and meteorology [43], [44].

In view of the state-of-the-art described above, the contributions of this paper are threefold:

- We model electricity market mechanisms using a Hidden Markov Model (HMM). The net aggregate supply curve is modeled as the hidden states of the system, and spot 
prices and total electricity traded as the observed states [30], [45], [46]. The hidden states and observations are modeled as random variables and the HMM provides a graphical representation of the conditional dependences between these variables. This model provides a natural framework for a Bayesian inference approach.

- We present a novel algorithm to approximate the posterior distribution of the aggregate supply curve in a dayahead electricity market, conditionally on observations on spot prices and total electricity traded. The proposed algorithm relies on classical MCMC and SMC algorithms developed in the literature [47]. Our main contribution is to introduce a simulation-based SMC approximation of the traditional Gibbs sampler algorithm.

- We show the accuracy of the proposed algorithm on a modified version of the IEEE 24-bus system. As information on real supply curves is not available, we randomly generate offering bids and clear the day-ahead market over the thirty-day simulation period. The market outcomes are used as inputs for the inference problem. This way we can test the proposed algorithm in a controlled environment and compare the outputs to the simulated supply curves.

The structure of this paper is the following. Section II introduces the problem and its formulation as a Bayesian inference problem. The proposed Bayesian inference algorithm is detailed in Section III. In Section IV we implement the proposed algorithm on a modified version of the IEEE 24-bus system and discuss the results. Finally, Section V concludes the paper and gathers perspectives regarding future works.

\section{INFERENCE PROBLEM FORMULATION}

In this section, we introduce a novel HMM formulation of a market clearing mechanism and we formulate mathematically the supply curve inference problem. The proposed inference algorithm and its implementation are further detailed in Sections III and IV.

\section{A. Electricity Market Clearing Mechanism}

We consider a strategic producer participating in a dayahead electricity market. We propose a market clearing model similar to European electricity markets. Participants submit their offers to the market operator for each hour of the following day. They can place two types of offers: single hourly or block orders, linking different hours of the day. As a large majority of the energy is traded through single hourly orders we neglect block orders [48]. For single hourly orders, each participant bids a combination of prices and quantities, represented by its supply function $S F_{g, d, h}\left(P_{g, d, h}\right)$. In oligopolistic markets, strategic producers can increase their profit by bidding a supply function that deviates form their true marginal cost. We adopt a Linear Supply Function Equilibrium (LSFE) model [49], such that

$$
S F_{g, d, h}\left(P_{g, d, h}\right)=\alpha_{g, d, h} P_{g, d, h}+\beta_{g, d, h} .
$$

Wind producers are assumed to offer their production at zero marginal cost. Finally, producers can exercise their strategic behavior by curtailing some of their production, i.e. by altering their offered quantity $\bar{P}_{g, d, h}$.

Since in practice the competition on the demand side is fairly limited, we assume a completely inelastic demand. It is important to note that this market modeling assumption does not result in any loss of generality in the proposed inference algorithm. Indeed, we are only interested in inferring the aggregate supply curve based on observations on spot prices and total electricity traded. These observations can be thought of as the intersection points between the demand and supply curves. Hence, assumptions on the shape of the demand curve do not influence the inference algorithm.

Finally, we neglect transmission constraints, ramping limits and unit commitment variables because we focus on a European market. As a result, the market clearing for a specific day $d$ can be formulated as

$$
\begin{array}{lll}
\min _{\Omega_{d}} & \sum_{g, h}\left(\alpha_{g, d, h} P_{g, d, h}+\beta_{g, d, h}\right) P_{g, d, h} & \\
\text { s.t. } & \sum_{g} P_{g, d, h}+\sum_{w} P_{w, d, h}=L_{d, h} & \forall h \quad: \lambda_{d, h}^{s p o t} \\
& 0 \leq P_{g, d, h} \leq \bar{P}_{g, d, h} & \forall g, h \\
& 0 \leq P_{w, d, h} \leq \bar{P}_{w, d, h} & \forall w, h
\end{array}
$$

where $\Omega_{d}=\left\{P_{g, d, h}, P_{w, d, h}: \forall g, w, h\right\}$. The objective of this convex optimization problem is to minimize the production cost in (2a), subject to power balance equations (2b), and power output limits (2c)-(2d). As a result, the spot prices $\lambda_{d, h}^{s p o t}$ are the dual variables of the power balance equations.

\section{B. Bayesian Inference Framework}

The supply functions of individual participants in the dayahead electricity market and the resulting aggregate supply curve are not usually disclosed by the market operator. Our aim is to reveal the historic aggregate supply curve, based on publicly available information. In practice market data may vary depending on the transparency policies of specific market operators. For instance, in [29] it is assumed that spot prices and dispatched production of each market participant can be observed. However, this assumption limits the applicability of the proposed method. That is why we consider that only spot prices and total electricity traded are disclosed. The market clearing mechanism described in Section II-A can be modeled as a factorial HMM. In this approach the parameters of the net aggregate supply curve, such as price parameters $\alpha_{g, d, h}$ and $\beta_{g, d, h}$ or offered production $\bar{P}_{g, d, h}$, can be modeled as the hidden states of the system, and the spot prices and total electricity traded as the observed states [30], [45], [46].

We can distinguish two applications. In the most general case, no information is available on individual market participants. We can then adopt a LSFE model for the aggregate supply curve, such that

$$
S F_{b, d, h}\left(P_{b, d, h}\right)=\alpha_{b, d, h} P_{b, d, h}+\beta_{b, d, h} \forall b .
$$

Note that the generation blocks $b$ are of arbitrary size (e.g. $10 \mathrm{MW}$ ) and do not necessarily represent individual market participants. As a result, we can model the price parameters of the aggregate supply function, $\alpha_{b, d, h}$ and $\beta_{b, d, h}$, as 
hidden variables. Furthermore, if more information on the technical characteristics of market participants is available, such as maximum power output and ramping limits, we can model the price parameters of the individual supply function $S F_{g, d, h}\left(P_{g, d, h}\right)$ and the offered production $\bar{P}_{g, d, h}$ as hidden variables. However, this is not included in the scope of our study.

We consider for now that no information on individual generators is available. Fig. 1 shows the structure of the proposed HMM. Each generation block $b$ fixes its price

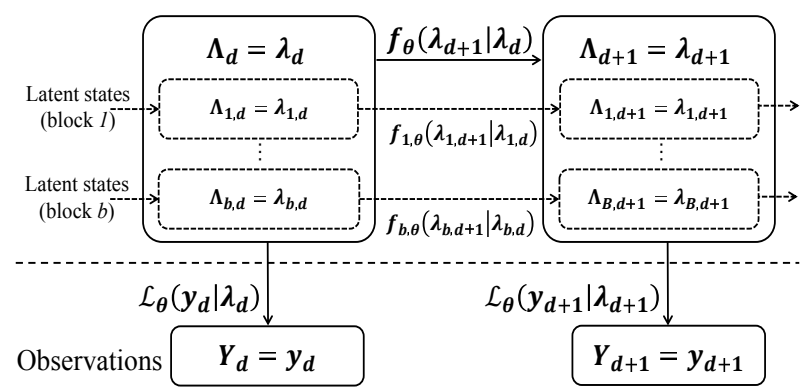

Fig. 1. HMM structure of the market clearing mechanism

parameters vector $\lambda_{b, d}=\left[\alpha_{b, d, h}, \beta_{b, d, h}: h=1, \ldots, 24\right]$ for a specific day $d$. The hidden variables $\lambda_{b, d}$ are modeled as random vectors, denoted $\Lambda_{b, d}$. Each generation block $b$ updates its offer from one day to the following, using a so-called introspection process. It is not required for us to know the nature of this introspection process. We model the stochastic process $\Lambda_{b, 1: D}=\left\{\Lambda_{b, d}: d=1, \ldots, D\right\}$ as a first-order Markov process. Our prior knowledge of the latent states and the introspection process is summarized by the initial density

$$
\mu_{b, \theta}\left(\lambda_{b, 1}\right)=\mathbb{P}\left(\Lambda_{b, 1}=\lambda_{b, 1} \mid \Theta=\theta\right),
$$

and transition function

$$
\begin{aligned}
& f_{b, \theta}\left(\lambda_{b, d+1} \mid \lambda_{b, d}\right) \\
& =\mathbb{P}\left(\Lambda_{b, d+1}=\lambda_{b, d+1} \mid \Lambda_{b, d}=\lambda_{b, d}, \Theta=\theta\right) .
\end{aligned}
$$

The transition function and initial density also depend on a set of static model parameters. These parameters are also assumed unknown and modeled as a random vector $\Theta$. The prior density $\pi(\theta)=\mathbb{P}(\Theta=\theta)$ represents our prior knowledge of these parameters.

As we are interested in inferring the aggregate supply curve rather than the individual supply curves of rival participants, we consider directly the collated random vectors $\Lambda_{d}=\left[\Lambda_{b, d}: b=1, \ldots, B\right]$ and the stochastic process $\Lambda_{1: D}=$ $\left\{\Lambda_{d}: d=1, \ldots, D\right\}$, taking values in $\Phi \subseteq \mathbb{R}^{2 H B D}$, modeling the aggregate latent states of all generation blocks. As participants cannot observe the bids of their rivals, the stochastic processes $\Lambda_{b, 1: D}$ are assumed mutually independent. As a result, the stochastic process $\Lambda_{1: D}$ is a Markov process and our prior knowledge of the aggregate latent states is summarized by the initial density and transition function

$$
\left\{\begin{array}{l}
\mu_{\theta}\left(\lambda_{1}\right)=\prod_{b} \mu_{b, \theta}\left(\lambda_{b, 1}\right), \\
f_{\theta}\left(\lambda_{d+1} \mid \lambda_{d}\right)=\prod_{b} f_{b, \theta}\left(\lambda_{b, d+1} \mid \lambda_{b, d}\right) .
\end{array}\right.
$$

The realizations of the stochastic process $\Lambda_{1: D}$ can not be observed directly, however we can refine our prior knowledge through observations of the spot prices and electricity loads, denoted for simplicity $y_{d}=\left[\lambda_{d, h}^{s p o t}, L_{d, h}: h=1, \ldots, 24\right]$. In HMMs these observed states are also modeled as random vectors $Y_{d}$ and assumed conditionally independent given the latent states. Hence, the observations' likelihood can be expressed as

$$
\begin{aligned}
& \mathcal{L}_{\theta}\left(y_{d} \mid \lambda_{d}\right) \\
& =\mathbb{P}\left(Y_{d}=y_{d} \mid \Lambda_{1: D}=\lambda_{1: D}, Y_{1: d-1}=y_{1: d-1}, \Theta=\theta\right) \\
& =\mathbb{P}\left(Y_{d}=y_{d} \mid \Lambda_{d}=\lambda_{d}, \Theta=\theta\right)
\end{aligned}
$$

Our aim is to infer the posterior density of the latent states and parameters conditionally on the observations over the selected period $d=1, \ldots, D$,

$$
p\left(\lambda_{1: D}, \theta \mid y_{1: D}\right)=\mathbb{P}\left(\Lambda_{1: D}=\lambda_{1: D}, \Theta=\theta \mid Y_{1: D}=y_{1: D}\right) .
$$

\section{BAYESIAN INFERENCE AlgORITHM}

In this section, we propose a novel states and parameters inference algorithm, targeting the posterior density of the aggregate supply curve by iterative sampling. We first present in Section III-A a general Gibbs sampler algorithm. We then introduce in Section III-B a novel simulation-based SMC approximation of the tradition Gibbs sampler and detail the proposed algorithm in Section III-C. The implementation of thi algorithm is further detailed in Section IV-B.

\section{A. Gibbs Sampler}

As the target density $p\left(\lambda_{1: D}, \theta \mid y_{1: D}\right)$ is unknown, it is not possible to sample directly from it. But Bayes' formula provides an expression of the posterior density in function of the observations' likelihood and prior density, up to a normalizing constant,

$$
\begin{aligned}
& p\left(\lambda_{1: D}, \theta \mid y_{1: D}\right) \\
& \propto \pi(\theta) \mu_{\theta}\left(\lambda_{1}\right) \prod_{d=1}^{D} \mathcal{L}_{\theta}\left(y_{d} \mid \lambda_{d}\right) f_{\theta}\left(\lambda_{d+1} \mid \lambda_{d}\right),
\end{aligned}
$$

where $\propto$ represents the proportionality operator. In that case, Markov Chain Monte Carlo (MCMC) methods allow us to approximate this target density by generating a correlated sequence of samples $\left\{\lambda_{1: D}^{(m)}, \theta^{(m)}: m=1, \ldots, M\right\}$ using a Markov process [32]-[34]. That is, at each iteration $(m+1)$ the updated values are drawn from a Markov transition kernel $K\left(\lambda_{1: D}^{(m+1)}, \theta^{(m+1)} \mid \lambda_{1: D}^{(m)}, \theta^{(m)}\right)$, from which it is easy to sample. If this transition kernel satisfies the detailed balance condition, the Markov chain will admit the target density as stationary distribution [34]. Hence, after a transient phase, the realized states $\left\{\lambda_{1: D}^{(m)}, \theta^{(m)}: m=M_{0}, \ldots, M\right\}$ will mimic samples drawn from the target density.

MCMC methods mainly rely on the Metropolis-Hastings (MH) algorithm [50]. At iteration $(m+1)$ the $\mathrm{MH}$ update 
involves drawing a candidate value from a proposal density $q\left(\lambda_{1: D}^{*}, \theta^{*} \mid \lambda_{1: D}^{(m)}, \theta^{(m)}\right)$. The candidate is accepted with probability

$$
\begin{aligned}
& \alpha\left(\lambda_{1: D}^{*}, \theta^{*} \mid \lambda_{1: D}^{(m)}, \theta^{(m)}\right) \\
& =\min \left[1, \frac{p\left(\lambda_{1: D}^{*}, \theta^{*} \mid y_{1: D}\right) q\left(\lambda_{1: D}^{(m)}, \theta^{(m)} \mid \lambda_{1: D}^{*}, \theta^{*}\right)}{p\left(\lambda_{1: D}^{(m)}, \theta^{(m)} \mid y_{1: D}\right) q\left(\lambda_{1: D}^{*}, \theta^{*} \mid \lambda_{1: D}^{(m)}, \theta^{(m)}\right)}\right],
\end{aligned}
$$

otherwise the Markov chain remains at $\lambda_{1: D}^{(m+1)}=\lambda_{1: D}^{(m)}$ and $\theta^{(m+1)}=\theta^{(m)}$. Although the target density is unknown, based on Bayes' formula (9) we can express it up to a normalizing constant and compute the acceptance ratio in (10). By construction the transition kernel of this Markov chain satisfies the detailed balance equation and the $\mathrm{MH}$ algorithm admits $p\left(\lambda_{1: D}, \theta \mid y_{1: D}\right)$ as stationary distribution. As a result, under weak assumptions on the proposal density, asymptotic convergence is guaranteed [34].

However, in high-dimensional problems such as this one, designing an efficient proposal density function is a difficult task. For that reason, we implement the Gibbs sampler algorithm. It is a special case of the $\mathrm{MH}$ algorithm, for which the parameters and latent states are updated alternatively using their posterior densities as proposal densities [51], such that

$$
\begin{aligned}
& \theta^{(m)} \sim p\left(. \mid \lambda_{1: D}^{(m-1)}, y_{1: D}\right), \\
& \lambda_{1: D}^{(m)} \sim p\left(. \mid y_{1: D}, \theta^{(m)}\right) .
\end{aligned}
$$

It results from (10) that the acceptance probability in the Gibbs sampler is always equal to one.

The Gibbs sampler is a $\mathcal{O}\left(d^{\Phi}\right)$ algorithm, which can become computationally challenging when the dimension of the problem $\left(d^{\Phi}=2 B D H\right)$ grows [35]. Finally, in most cases it is easy to sample directly from $p\left(\theta \mid \lambda_{1: D}^{(m-1)}, y_{1: D}\right)$. Assuming that we can sample from $p\left(\lambda_{1: D} \mid y_{1: D}, \theta^{(m)}\right)$, the Gibbs sampler algorithm builds iteratively a sequence of samples $\left\{\lambda_{1: D}^{(m)}, \theta^{(m)}: m=M_{0}, \ldots, M\right\}$ approximately distributed according to the target density.

\section{B. Sequential Monte Carlo Approximation}

For a given value $\theta^{(m)}$ of the parameters it is generally not possible to sample directly from the posterior density $p\left(\lambda_{1: D} \mid y_{1: D}, \theta^{(m)}\right)$. The authors in [47] suggest approximating it using a Sequential Monte Carlo (SMC) algorithm. The general idea behind SMC algorithms is to build iteratively a large cloud of equally-weighted particles $\left\{\lambda_{1: D}^{(i)}: i=1, \ldots, N\right\}$ approximately distributed according to the posterior density $p\left(\lambda_{1: D} \mid y_{1: D}, \theta^{(m)}\right)$ [37]-[40].

For that, we divide the task by sequentially approximating the densities $p\left(\lambda_{1: d} \mid y_{1: d}, \theta^{(m)}\right)$ for $d=1, \ldots, D$. At each step $d$, we consider the cloud of equally-weighted particles $\left\{\lambda_{1: d}^{(i)}: i=1, \ldots, N\right\}$ approximately distributed according to the density of interest $p\left(\lambda_{1: d} \mid y_{1: d}, \theta^{(m)}\right)$. The factorization

$$
\begin{aligned}
& p\left(\lambda_{1: d+1} \mid y_{1: d+1}, \theta^{(m)}\right) \\
& \propto p\left(\lambda_{1: d} \mid y_{1: d}, \theta^{(m)}\right) f_{\theta^{(m)}}\left(\lambda_{d+1} \mid \lambda_{d}\right) \mathcal{L}_{\theta^{(m)}}\left(y_{d+1} \mid \lambda_{d+1}\right)
\end{aligned}
$$

suggests propagating each particle $\lambda_{1: d}^{(i)}$ to the following day using an importance sampling-resampling mechanism. $N$ offspring particles $\tilde{\lambda}_{d+1}^{(i)}$ are sampled from a so-called importance function $q\left(\tilde{\lambda}_{d+1}^{(i)} \mid \lambda_{d}^{(i)}, y_{d+1}, \theta^{(m)}\right)$, and then resampled based on their relative importance weights,

$$
\begin{aligned}
w_{d+1}^{(i)} & \propto \frac{p\left(\lambda_{1: d}^{(i)}, \tilde{\lambda}_{d+1}^{(i)}, y_{1: d+1} \mid \theta^{(m)}\right)}{p\left(\lambda_{1: d}^{(i)}, y_{1: d} \mid \theta^{(m)}\right) q\left(\tilde{\lambda}_{d+1}^{(i)} \mid \lambda_{d}^{(i)}, y_{d+1}, \theta^{(m)}\right)} \\
& \propto \frac{f_{\theta^{(m)}}\left(\tilde{\lambda}_{d+1}^{(i)} \mid \lambda_{d}^{(i)}\right) \mathcal{L}_{\theta^{(m)}}\left(y_{d+1} \mid \tilde{\lambda}_{d+1}^{(i)}\right)}{q\left(\tilde{\lambda}_{d+1}^{(i)} \mid \lambda_{d}^{(i)}, y_{d+1}, \theta^{(m)}\right)} .
\end{aligned}
$$

At the resampling step, $N$ particles $\lambda_{d+1}^{(i)}$ are drawn from the weighted sample $\left\{\tilde{\lambda}_{d+1}^{(i)}: i=1, \ldots, N\right\}$. The resampling mechanism implemented in this study is further detailed in Appendix A. The resampling step is a bottleneck of the SMC algorithm but the authors in [52] proposed an efficient stratified resampling algorithm that takes $\mathcal{O}(N)$.

The resampled cloud of $N$ equally-weighted particles $\left\{\lambda_{1: d+1}^{(i)}: i=1, \ldots, N\right\}$ is now considered approximately distributed according to the probability density $p\left(\lambda_{1: d+1} \mid y_{1: d+1}, \theta^{(m)}\right)$ and propagated to the following step. The appeal of this approach is that it provides an estimator of the posterior density from which it is easy to sample,

$$
\hat{p}\left(\lambda_{1: D} \mid y_{1: D}, \theta^{(m)}\right)=\frac{1}{N} \sum_{i=1}^{N} \delta\left(\lambda_{1: D}^{(i)}-\lambda_{1: D}\right),
$$

where $\delta($.$) represents the Dirac delta function.$

However, Ref. [47] showed that an approximate Gibbs sampler, updating the states $\lambda_{1: D}^{(m+1)}$ by sampling from this Monte Carlo estimator does not admit the target density $p\left(\lambda_{1: D}, \theta \mid y_{1: D}\right)$ as stationary distribution. Instead, it is necessary to use a conditional SMC algorithm to approximate the posterior density $p\left(\lambda_{1: D} \mid y_{1: D}, \theta^{(m)}\right)$ at each iteration $m \geq 1$. The particles $\left\{\lambda_{1: D}^{(i)}: i=1, \ldots, N\right\}$ are generated conditionally on the reference trajectory $\lambda_{1: D}^{(m-1)}$, associated with the ancestral lineage $A_{1: D}^{m-1}$. The conditioning on the reference trajectory is implemented by ensuring that this path survives all the sampling and resampling steps and generating the remaining $N-1$ particles the usual way. Informally, the introduction of a reference trajectory can be thought of as guiding the sampled particles to a relevant region of the space. In practice, it ensures that the transition kernel leaves the target distribution invariant for any number of particles $N \geq 2$ [53].

Additionally, the importance function has a great influence on the accuracy of SMC algorithms. It is usually recommended 
to set the posterior density $p\left(\lambda_{d+1} \mid \lambda_{d}, y_{d+1}, \theta^{(m)}\right)$ as importance function [47]. As it is not possible to sample directly from the posterior density, we use the transition function of the latent Markov chain $f_{\theta^{(m)}}\left(\tilde{\lambda}_{d+1} \mid \lambda_{d}\right)$ as importance function. As a result, the importance weights defined in (13) only depend on the observations' likelihood

$$
w_{d+1}^{(i)} \propto \mathcal{L}_{\theta^{(m)}}\left(y_{d+1} \mid \tilde{\lambda}_{d+1}^{(i)}\right) .
$$

This method relies on the assumption that the observations' likelihood $\mathcal{L}_{\theta^{(m)}}$ is known and can be computed pointwise. In our model $\mathcal{L}_{\theta^{(m)}}$ is difficult to express analytically due to the complex HMM structure. And designing a very uninformative likelihood function would reduce the efficiency of the resampling step in the SMC algorithm. Another solution, inspired by [43], [44], is to simulate for each particle $\tilde{\lambda}_{d+1}^{(i)}$ a theoretical market outcome $y_{d+1}^{(i)}=\left[\lambda_{d+1, h}^{s p o t(i)}, L_{d+1, h}^{(i)}: \forall w, g, h\right]$ using the market clearing model in Section II-A. This way, we can define the observations' likelihood and importance weights in function of the simulated markets outcomes. Additionally, since power balance $L_{d+1, h}^{(i)}=L_{d+1, h}$ is enforced for each particle, we can express the importance weights only in function of the observed and simulated spot prices without loss of generality, such that

$$
w_{d+1}^{(i)} \propto \mathcal{L}_{\theta^{(m)}}\left(\lambda_{d+1}^{s p o t} \mid \lambda_{d+1}^{s p o t(i)}\right) .
$$

The conditional SMC algorithm described above is detailed in Algorithm 1.

\section{Particle Gibbs Sampler}

Based on the Gibbs sampler and the conditional SMC algorithm described above, we propose a so-called Particle Gibbs Sampler (PGS) targeting the joint posterior density $p\left(\lambda_{1: D}, \theta \mid y_{1: D}\right)$. At each iteration $m \geq 1$, the parameters are updated using their posterior density $p\left(\theta^{(m)} \mid \lambda_{1: D}^{(m-1)}, y_{1: D}\right)$ as importance function. A conditional SMC algorithm (Algorithm 1) is run to generate a cloud of particles $\left\{\lambda_{1: D}^{(i)}: i=1, \ldots N\right\}$ approximately distributed according to the posterior density $p\left(\lambda_{1: D} \mid y_{1: D}, \theta^{(m)}\right)$. The latent states are updated by sampling from the Monte Carlo estimator defined in (14). In practice this is realized by sampling an index $i_{0}$ from the discrete uniform distribution $\mathcal{U}\{1, N\}$ and setting the states $\lambda_{1: D}^{(m)}=\lambda_{1: D}^{(i 0)}$, associated with the ancestral lineage $A_{1: D}^{m}=A_{1: D}^{i_{0}}$. The PGS described above is detailed in Algorithm 2.

\section{Case Study: Inferring the Aggregate Supply CURVE}

Data on rival participants' real supply functions is not available. As a result we build the case study by simulating rival participants bids and day-ahead market clearing over thirty days. That way, we can apply the proposed inference method in a controlled environment and assess its performance. Based on the observations on spot prices and total electricity traded, we try to infer the aggregate supply curve of this system. We then compare the outputs of the inference algorithm to the assumed data.
$\overline{\text { Algorithm } 1 \text { Conditional SMC algorithm at iteration } m \geq 1}$ Let $\lambda_{1: D}^{(m-1)}$ be the reference path, associated with the ancestral lineage $A_{1: D}^{m-1}$

for $d=1$ do

For $i \neq A_{1}^{m-1}$, sample the $N-1$ particles $\tilde{\lambda}_{1}^{(i)} \sim$ $\mu_{\theta(m)}($.

For $i=A_{1}^{m-1}$, set the particle $\tilde{\lambda}_{1}^{(i)}=\lambda_{1}^{(m-1)}$

Simulate a market clearing for each particle $\tilde{\lambda}_{1}^{(i)}$ and compute the theoretical market outcomes $y_{1}^{(i)}$

Compute the importance weights $w_{1}^{(i)}$ for all particles

For $i \neq A_{1}^{m-1}$, resample the $N-1$ particles $\lambda_{1}^{(i)}$ and their ancestral indexes $A_{1}^{i}$ from the weighted sample $\left\{\tilde{\lambda}_{1}^{(i)}: i=1, \ldots, N\right\}$

For $i=A_{1}^{m-1}$, set the resampled particle $\lambda_{1}^{(i)}=\lambda_{1}^{(m-1)}$ and $A_{1}^{i}=A_{1}^{m-1}$

\section{end for}

for $d=2, \ldots, D$ do

For $i \neq A_{d}^{m-1}$, sample the $N-1$ particles $\tilde{\lambda}_{d}^{(i)} \sim$ $f_{\theta(m)}\left(\cdot \mid \lambda_{d-1}^{(i)}\right)$

For $i=A_{d}^{m-1}$, set the particle $\tilde{\lambda}_{d}^{(i)}=\lambda_{d}^{(m-1)}$

Simulate a market clearing for each particle $\tilde{\lambda}_{d}^{(i)}$ and compute the theoretical market outcomes $y_{d}^{(i)}$

Compute the importance weights $w_{d}^{(i)}$ for all particles

For $i \neq A_{d}^{m-1}$, resample the $N-1$ particles $\lambda_{d}^{(i)}$ and their ancestral indexes $A_{d}^{i}$ from the weighted sample $\left\{\tilde{\lambda}_{d}^{(i)}: i=d, \ldots, N\right\}$

For $i=A_{d}^{m-1}$, set the resampled particle $\lambda_{d}^{\left(B_{d}\right)}=$ $\lambda_{d}^{(m-1)}$ and $A_{d}^{i}=A_{d}^{m-1}$

Set $\lambda_{1: d}^{(i)}=\left\{\lambda_{1: d-1}^{\left(A_{d}^{i}\right)}, \lambda_{d}^{(i)}\right\}$ and $A_{1: d}^{i}=\left\{A_{1: d-1}^{A_{d}^{i}}, A_{d}^{i}\right\}$ end for 


\section{A. Case Study Setup}

We consider a modified version of the IEEE 24-bus system as presented in [54], with twelve thermal generators, and six wind farms of $200 \mathrm{MW}$ each. The technical characteristics of the participants are collated in Table I in Appendix B. We generate randomly the supply curves of each market participant by sampling for each hour of the thirty-day simulation period from the normal distributions

$$
\left\{\begin{aligned}
\alpha_{g, d, h} & \sim \mathcal{N}\left(\mu_{\alpha_{g}^{0}}, \sigma_{\alpha_{g}^{0}}^{2}\right) \forall d, h, \\
\beta_{g, d, h} & \sim \mathcal{N}\left(\mu_{\beta_{g}^{0}}, \sigma_{\beta_{g}^{0}}^{2}\right) \forall d, h .
\end{aligned}\right.
$$

The mean values and standard deviations of these Normal distributions are collated in Table I in Appendix B. Fig. 2 shows the simulated price parameters for each participant, as well as the median, upper and lower quartiles. In addition, we

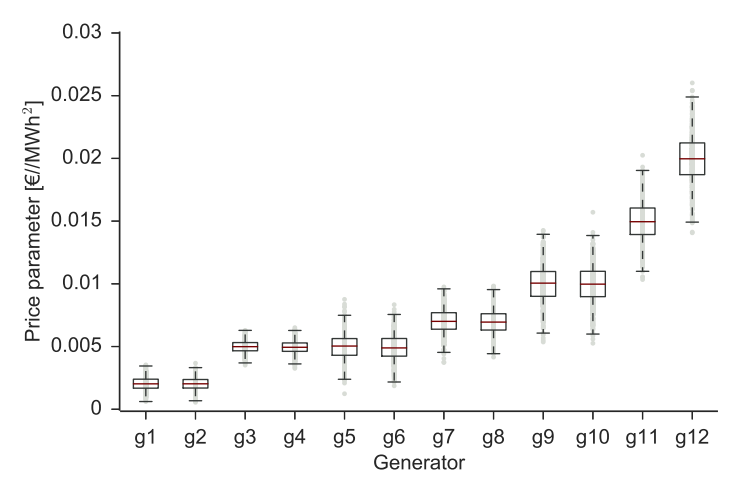

(a) Price parameters $\alpha_{g, d, h}$ bids (median, upper and lower quartiles)

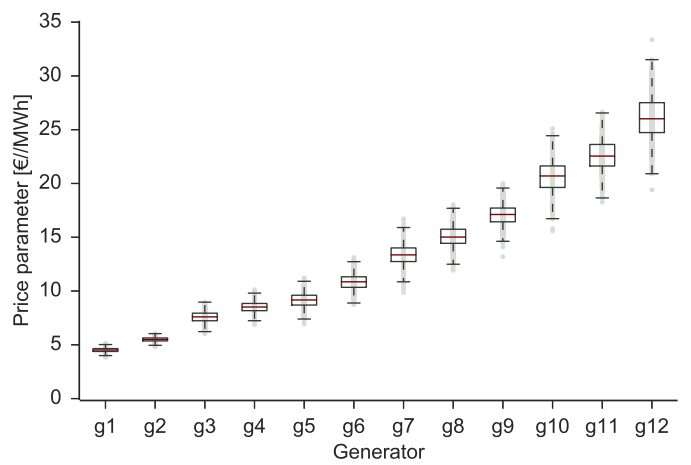

(b) Price parameters $\beta_{g, d, h}$ bids (median, upper and lower quartiles)

Fig. 2. Case Study Setup: Price parameters bids over the simulation period (30 days)

consider that market participants are not strategic in quantities, and offer their maximum available production, such that

$$
\bar{P}_{g, d, h+1}=\min \left\{\bar{P}_{g, d, h}+\bar{R}_{g}, \bar{P}_{g}\right\} \forall d, h .
$$

Fig. 3 shows the resulting aggregate supply curves for each hour of the simulation period.

We use the load profile data provided in [54] and introduce load factors for three representative days: base day, shoulder

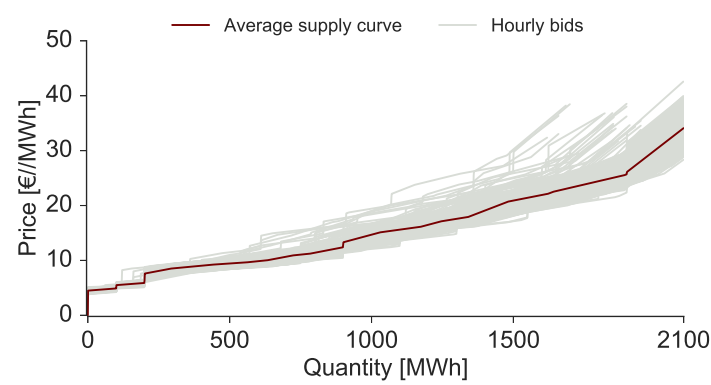

Fig. 3. [Simulated hourly aggregate supply curves and average supply curve over the simulation period (30 days)

day and peak day and generate randomly the load profiles for thirty days. As we assumed a European market framework without transmission constraints, we can aggregate the loads at each bus. In addition, we use historic wind production factors from Nord Pool. Finally, using this data we simulate a market clearing over thirty days. Fig. 4 shows the load profiles, total wind production, and spot prices over the simulation period.

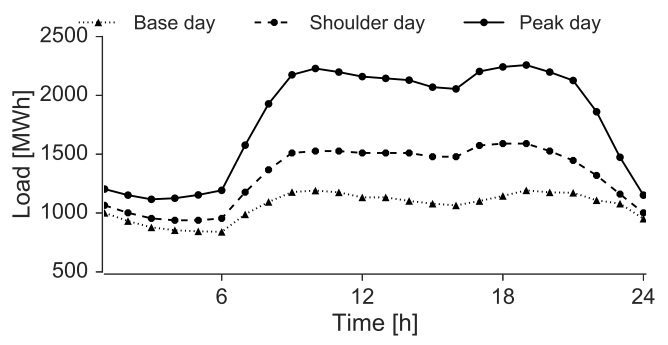

(a) Hourly electricity load for 3 representative days

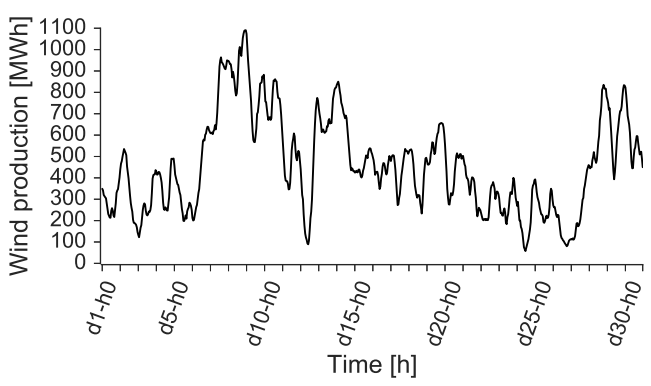

(b) Total hourly wind production over the simulation period (30 days)

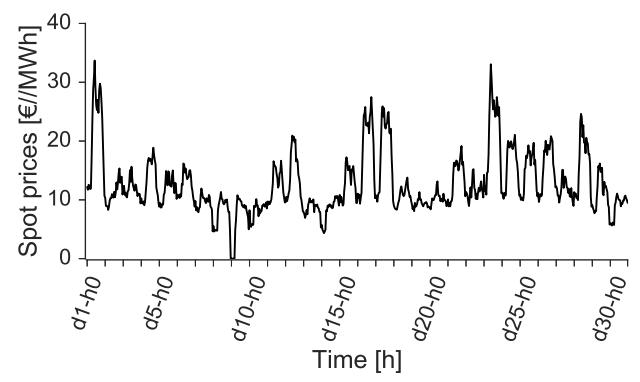

(c) Spot prices over the simulation period (30 days)

Fig. 4. Case Study Setup: Market clearing simulation results 


\section{B. Implementation of the PGS Algorithm}

We now assume that spot prices and total electricity traded on the market for each hour of the simulation period are directly available from market data. In addition, wind production over the simulation period can easily be estimated using historical meteorological data. Our goal is to estimate the posterior distribution of the aggregate supply curve, divided into $B=10$ generation blocks of $210 \mathrm{MW}$ each.

Our prior knowledge on the latent states $\alpha_{b, 1: D}$ and $\beta_{b, 1: D}$ is characterized by their prior densities. There is no systematic approach in the literature to select prior densities. When no information is available, it is recommended to set an uninformative prior density that covers the whole range of possible values. For each generation block $b$, we model the initial density and transition function of the Markov chain $\alpha_{b, 1: D}$ as independent Normal distributions

$$
\left\{\begin{array}{l}
\alpha_{b, 1} \mid \mu_{\alpha_{b}}, \sigma_{\alpha_{b}} \sim \mathcal{N}\left(M_{\alpha_{b}}, \Sigma_{\alpha_{b}}\right), \\
\alpha_{b, d+1} \mid \alpha_{b, d}, \sigma_{\alpha_{b}} \sim \mathcal{N}\left(\alpha_{b, d}, \Sigma_{\alpha_{b}}\right) \forall d
\end{array}\right.
$$

where $\Sigma_{\alpha_{b}}=\sigma_{\alpha_{b}}^{2} I_{24}$ is the covariance matrix and $M_{\alpha_{b}}=$ $\left[\mu_{\alpha_{b}}: h=1, \ldots, 24\right]$ the mean vector. In addition, the static parameters $\mu_{\alpha_{b}}$ and $h_{\alpha_{b}}=\frac{1}{\sigma_{\alpha_{b}}^{2}}$ are also considered unknown. A standard scheme in the literature is to use an independent Normal-Gamma prior to describe them, such that

$$
\left\{\begin{array}{l}
\mu_{\alpha_{b}} \sim \mathcal{N}\left(m_{\alpha_{b}}, V_{\alpha_{b}}\right), \\
h_{\alpha_{b}} \sim \Gamma\left(a_{\alpha_{b}}, b_{\alpha_{b}}\right),
\end{array}\right.
$$

where $a_{\alpha_{b}}$ is the shape parameter and $b_{\alpha_{b}}$ the inverse scale parameter. By analogy, we define Normal initial densities and transition functions for the latent states $\beta_{b, 1: D}$, and NormalGamma prior densities for the static model parameters $\mu_{\beta_{b}}$ and $h_{\beta_{b}}$.

We select the hyperparameters of the prior densities

$m_{\beta}=[10.0,11.7,13.3,15.0,16.7,18.3,20.0,21.7,23.3,25.0]$, $m_{\alpha_{b}}=0.001, V_{\alpha_{b}}=0.001, V_{\beta_{b}}=100, a_{\beta_{b}}=b_{\beta_{b}}=10^{-4}$, $a_{\alpha_{b}}=0.1$ and $b_{\alpha_{b}}=5$ for all the generation blocks. Fig. 5 shows the initial guess on the average aggregate supply curve, such that $S F_{b}\left(P_{b}\right)=m_{\alpha_{b}} P_{b}^{2}+m_{\beta_{b}} P_{b}$.

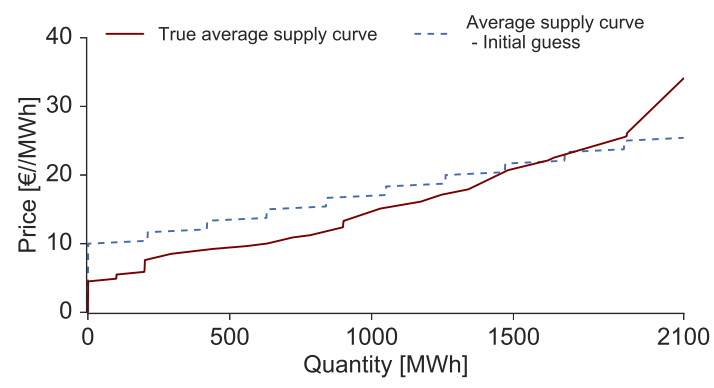

Fig. 5. Initial guess on the average supply curve: $S F_{b}\left(P_{b}\right)=m_{\alpha_{b}} P_{b}^{2}+$ $m_{\beta_{b}} P_{b}$

As explained in Section III-B, the observations' likelihood is defined in function of the simulated spot prices $\hat{\lambda}_{d}^{\text {spot }(i)}$. For simplicity we assume a Normal distribution

$$
\lambda_{d}^{\text {spot }} \mid \lambda_{d}^{\text {spot }(i)}, \Sigma^{\text {spot }} \sim \mathcal{N}\left(\lambda_{d}^{\text {spot }(i)}, \Sigma^{\text {spot }}\right)
$$

where $\Sigma^{s p o t}=\sigma^{s p o t^{2}} I_{24}$ is the covariance matrix. We set arbitrary $\sigma^{\text {spot }}=2$.

At each Gibbs iteration $m$, we update the static parameters' posterior densities based on the expression of the observations' likelihood and prior densities, such that

$$
\left\{\begin{array}{l}
\mu_{\alpha_{b}}^{(m)} \mid y_{1: D}, h_{\alpha_{b}}^{(m-1)} \sim \mathcal{N}\left(\bar{m}_{\alpha_{b}}, \bar{V}_{\alpha_{b}}\right) \\
h_{\alpha_{b}}^{(m)} \mid y_{1: D}, \mu_{\alpha_{b}}^{(m)} \sim \Gamma\left(\bar{a}_{\alpha_{b}}, \bar{b}_{\alpha_{b}}\right)
\end{array}\right.
$$

where

$$
\left\{\begin{array}{l}
\frac{1}{V_{\alpha_{b}}}=\frac{1}{V_{\alpha_{b}}}+h_{\alpha_{b}}^{(m-1)}, \\
\bar{m}_{\alpha_{b}}=\bar{V}_{\alpha_{b}}\left(\frac{m_{\alpha_{b}}}{V_{\alpha_{b}}}+h_{\alpha_{b}}^{(m-1)} \alpha_{b, 1}^{(m-1)}\right), \\
\bar{a}_{\alpha_{b}}=a_{\alpha_{b}}+\frac{D}{2}, \\
\bar{b}_{\alpha_{b}}=b_{\alpha_{b}}+\frac{\left(\alpha_{b, 1}^{(m-1)}-\mu_{\alpha_{b}}^{(m)}\right)^{2}+\sum_{t=2}^{T}\left(\alpha_{b, t}^{(m-1)}-\alpha_{b, t-1}^{(m-1)}\right)^{2}}{2} .
\end{array}\right.
$$

As suggested in the literature, an adequate number of particles for the SMC algorithm is $N=2000$. Although $\mathrm{MH}$ and Gibbs sampler algorithms are guarantied to converge asymptotically, at a given iteration $m$ there is no clear indication on whether they have converged. Providing tight bounds for the convergence rate of these algorithms can be challenging but the authors in [55]-[57] showed that under mild assumptions the convergence rate is polynomial in the dimension of the problem. Additionally, various diagnostic tools can be applied to the outputs of the algorithm to assess its convergence [58], [59]. As we implement the proposed PGS algorithm in a controlled environment, we empirically choose $M=200$ iterations to achieve convergence.

\section{Results}

We implemented this algorithm in Python 3.5, using Gurobi 7.0.2 solver, on a Processor Intel Core i5-6200U CPU @ 2.30 $\mathrm{GHz}$ (8.00 GB RAM). The CPU time for each SMC iteration is 9.15 seconds. The total execution time of the algorithm depends on the mixing rate of the Markov chain.

After a transient phase $\left(M_{0}=100\right)$ the correlated sequence $\left\{\lambda_{1: D}^{(m)}, \theta^{(m)}: m=M_{0}, \ldots, M\right\}$ is considered approximately distributed according to the target density $p\left(\lambda_{1: D}, \theta \mid y_{1: D}\right)$. Fig. 6 shows the MCMC states of the static parameters $\mu_{\alpha_{g}}^{(m)}$ and $\mu_{\beta_{g}}^{(m)}$ for $m=M_{0}, \ldots, M$. Additionally, the estimated values of the static price parameters are collated in Table II in Appendix B. These values can not directly be compared to the true price parameters of the participants. But, Fig. 7 shows the resulting estimated aggregate supply curve and the $2 \sigma$ confidence interval, based on the estimated static price parameters. This shows that the proposed algorithm is able to approximate the aggregate supply curve with no prior information on rival participants. However, few observations are available for net loads lower than $420 \mathrm{MWh}$ and higher than $1850 \mathrm{MWh}$, i.e. the market participants in this region of the aggregate supply curve are rarely marginal. As a result, these regions of the aggregtae supply curve can not be estimated accurately.

As previously discussed, the proposed algorithm provides accurate information on the historic aggregate supply curve, 


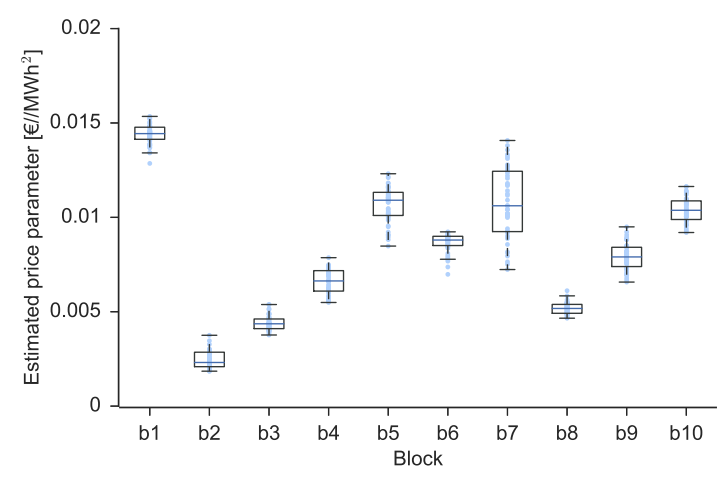

(a) Static price parameters $\mu_{\alpha_{g}}$

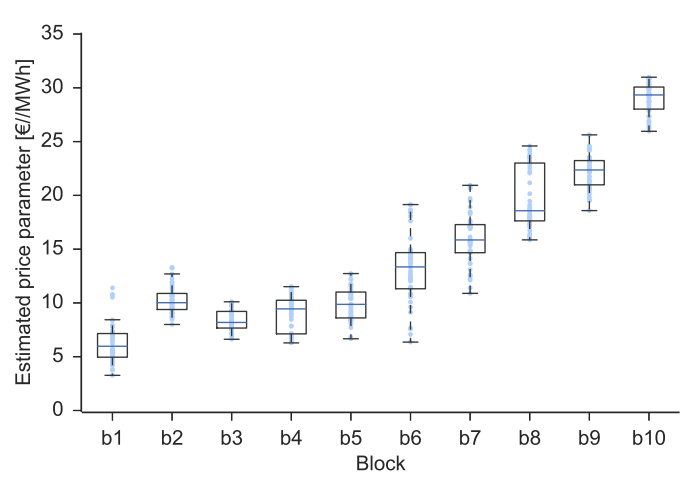

(b) Static price parameters $\mu_{\beta_{g}}$

Fig. 6. MCMC states of the static parameters (median, upper and lower quartiles)

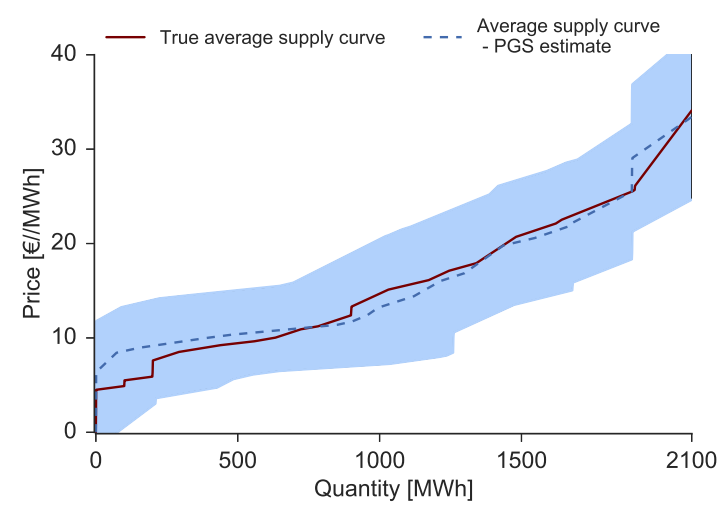

Fig. 7. Case study 1: Estimated aggregate supply curve and $2 \sigma$ confidence interval

which is a valuable input for supply function prediction tools relying on multi-agent-based models and neural networks [12], [17], [27]. Additionally, the accuracy of hierarchical optimization approaches presented in [19]-[21], [23], relies on the ability to generate accurate scenarios of the aggregate supply curve. As a result, the description of the uncertainty through its posterior density, is a key piece of information for strategic producers.

\section{Conclusion}

The Particle Gibbs Sampler algorithm proposed in this paper provides a complete model of the uncertainty of the aggregate supply curve, through an estimate of its posterior probability density. We showed on a modified version of the IEEE 24-bus system that this method allows us to accurately approximate the aggregate supply curve with no prior information on rival participants. These results provide valuable inputs for supply function prediction tools, and decision-making problems under uncertainty. Additionally, for regulators this approach can be useful to monitor market power and competition behaviors in electricity markets.

This work opens up various opportunities for future research. First, the model proposed in this paper can be generalized to accommodate different market frameworks by adapting the market clearing mechanism in Section II. In particular, it is possible to study unit commitment problems by including network and operational constraints. In that setting, the technical characteristics of the generators, can be treated as unknown static parameters and their posterior density can be estimated as well. Additionally, this study focuses on inferring the aggregate supply curve. A natural extension to this work is to estimate the individual supply curves of rival participants. Competition in quantity can be introduced by modeling quantities offered to the market as latent states.

Furthermore, as previously discussed, the computational complexity and convergence rates is a major limitation for implementing SMC and MCMC algorithms to high-dimensional inference problems. Ref. [32] presents various methods for improving the convergence rate of $\mathrm{MH}$ and Gibbs sampler algorithms. Additionally, certain steps of the algorithm, such as sampling and resampling, can easily be parallelized. Alternative inference methods could also be investigated to address this issue. It is sometime suggested that combining deterministic and stochastic inference techniques, also called Rao-Blackwellisation, can improve greatly the computational time of MCMC algorithms [60]. Similarly, methods combining variational approximation and MCMC could allow improving convergence speed of standard MCMC algorithms [61]. Finally, the method proposed in this paper can be generalized by investigating nonparametric Bayesian inference methods, allowing us to reduce the number of assumptions on the underlying states and parameters [62].

\section{Appendix A: Stratified Resampling Algorithm}

In order to mitigate the variance introduced by standard multinomial resampling, we implement a stratified resampling mechanism [63]. We sample $N$ independent random variables from the uniform distributions

$$
u^{(i)} \sim \mathcal{U}\left(\frac{i-1}{N}, \frac{i}{N}\right] \quad \text { for } \quad i=1, \ldots, N .
$$

We then define the ancestral indexes $A_{d+1}^{i}=j$ and the resampled particles $\lambda_{d+1}^{(i)}=\tilde{\lambda}_{d+1}^{(j)}$, such that

$$
\sum_{k=1}^{j-1} w_{d+1}^{(k)}<u^{(i)} \leq \sum_{k=1}^{j} w_{d+1}^{(k)} .
$$


As a result, this resampling mechanism allows the particles with the lowest importance weights to be resampled at most once.

\section{Appendix B: Case Study Detailed Parameters and RESULTS}

TABLE I

CASE STUDY SETUP: GENERATION UNITS PARAMETERS

\begin{tabular}{ccccccc}
\hline & $g_{1}$ & $g_{2}$ & $g_{3}$ & $g_{4}$ & $g_{5}$ & $g_{6}$ \\
\hline $\bar{P}_{g}$ & 100 & 100 & 200 & 200 & 300 & 300 \\
$\bar{R}_{g}$ & 20 & 20 & 60 & 80 & 80 & 180 \\
$\mu_{\beta_{g}^{0}}$ & 4.5 & 5.5 & 7.6 & 8.5 & 9.2 & 10.9 \\
$\sigma_{\beta_{g}^{0}}$ & 0.2 & 0.2 & 0.5 & 0.5 & 0.7 & 0.7 \\
$\mu_{\alpha_{g}^{0}}$ & 0.002 & 0.002 & 0.005 & 0.005 & 0.005 & 0.005 \\
$\sigma_{\alpha_{g}^{0}}$ & 0.0005 & 0.0005 & 0.0005 & 0.0005 & 0.001 & 0.001 \\
\hline & $g_{7}$ & $g_{8}$ & $g_{9}$ & $g_{10}$ & $g_{11}$ & $g_{12}$ \\
\hline $\bar{P}_{g}$ & 200 & 200 & 250 & 250 & 100 & 100 \\
$\bar{R}_{g}$ & 20 & 20 & 60 & 80 & 80 & 180 \\
$\mu_{\beta_{g}^{0}}$ & 13.3 & 15.1 & 17.1 & 20.7 & 22.5 & 26.1 \\
$\sigma_{\beta_{g}^{0}}$ & 1.0 & 1.0 & 1.0 & 1.5 & 1.5 & 2.0 \\
$\mu_{\alpha_{g}^{0}}$ & 0.007 & 0.007 & 0.01 & 0.01 & 0.015 & 0.02 \\
$\sigma_{\alpha_{g}^{0}}$ & 0.001 & 0.001 & 0.0015 & 0.0015 & 0.0015 & 0.002 \\
\hline
\end{tabular}

TABLE II

CASE STUDY: ESTIMATED POSTERIOR DENSITY OF THE AGGREGATE SUPPLY CURVE

\begin{tabular}{cccccc}
\hline & $b_{1}$ & $b_{2}$ & $b_{3}$ & $b_{4}$ & $b_{5}$ \\
\hline$\hat{\mu}_{\beta_{b}}$ & 6.35 & 10.33 & 8.43 & 8.93 & 9.92 \\
$\hat{\sigma}_{\beta_{b}}$ & 4.02 & 1.92 & 2.41 & 1.41 & 2.64 \\
$\hat{\mu}_{\alpha_{b}}$ & 0.01438 & 0.00246 & 0.00440 & 0.00660 & 0.01067 \\
$\hat{\sigma}_{\alpha_{g}}$ & 0.00154 & 0.00035 & 0.00088 & 0.00089 & 0.00138 \\
\hline \multicolumn{1}{c}{$b_{6}$} & $b_{7}$ & $b_{8}$ & $b_{9}$ & $b_{10}$ \\
\hline$\hat{\mu}_{\beta_{b}}$ & 13.19 & 16.01 & 19.79 & 22.20 & 29.05 \\
$\hat{\sigma}_{\beta_{b}}$ & 3.78 & 2.72 & 3.15 & 3.17 & 3.90 \\
$\hat{\mu}_{\alpha_{b}}$ & 0.00865 & 0.01077 & 0.00517 & 0.00792 & 0.01040 \\
$\hat{\sigma}_{\alpha_{b}}$ & 0.00095 & 0.00197 & 0.00071 & 0.00100 & 0.00116
\end{tabular}

\section{ACKNOWLEDGMENTS}

The authors acknowledge Dr. Robin Girard, for general input to this paper. Six reviewers, as well as an editor, are to be acknowledged for their comments and suggestions, which permitted to improve the version of the manuscript submitted originally.

\section{REFERENCES}

[1] T. Jamasb and M. Pollitt, "Electricity market reform in the european union: review of progress toward liberalization \& integration," The Energy Journal, pp. 11-41, 2005.

[2] T. Pham, "Market power in power markets in europe: the cases in french and german woholesale electricity markets," Ph.D. dissertation, Université Paris Dauphine-Paris IX, 2015.

[3] D. P. Brown and D. E. Olmstead, "Measuring market power and the efficiency of alberta's restructured electricity market: An energy-only market design," 2016.

[4] S. P. Karthikeyan, I. J. Raglend, and D. P. Kothari, "A review on market power in deregulated electricity market," International Journal of Electrical Power \& Energy Systems, vol. 48, pp. 139-147, 2013.

[5] F. P. Sioshansi, "Electricity market reform and'reform of the reforms'," Int. J. Global Energy Issues, vol. 29, no. 1-2, pp. 3-27, 2008
[6] N. Kroes (European Commissioner for Competition Policy), "More competitive energy markets: building on the findings of the sector inquiry to shape the right policy solutions," in SPEECH/07/547. European Energy Institute, Brussels, Sept. 2007.

[7] "Regulation (eu) no 1227/2011 on wholesale energy market integrity and transparency," in Official J. European Union. THE EUROPEAN PARLIAMENT AND THE COUNCIL OF THE EUROPEAN UNION, Oct. 2011.

[8] B. Bosco, L. Parisio, and M. Pelagatti, "Estimating marginal costs and market power in the italian electricity auctions," in 7th Int. Conf. European Energy Market (EEM). IEEE, 2010, pp. 1-6.

[9] A. Hortacsu and S. L. Puller, "Understanding strategic bidding in multiunit auctions: a case study of the texas electricity spot market," RAND J. Econ., vol. 39, no. 1, pp. 86-114, 2008.

[10] L. Baringo and A. J. Conejo, "Offering strategy via robust optimization," IEEE Trans. Power Syst., vol. 26, pp. 1418-1425, Aug. 2011.

[11] J. M. Morales, A. J. Conejo, and J. Pérez-Ruiz, "Short-term trading for a wind power producer," IEEE Trans. Power Syst., vol. 25, pp. 554-564, Feb. 2010.

[12] I. Praça, C. Ramos, Z. Vale, and M. Cordeiro, "Mascem: a multiagent system that simulates competitive electricity markets," IEEE Intell. Syst., vol. 18, no. 6, pp. 54-60, Nov. 2003.

[13] A. Weidlich and D. Veit, "A critical survey of agent-based wholesale electricity market models," Energy Econ., vol. 30, no. 4, pp. 1728-1759, July 2008.

[14] S. A. Gabriel, A. J. Conejo, J. D. Fuller, B. F. Hobbs, and C. Ruiz, Complementarity Modeling in Energy Markets. New York, NY, USA: Springer, 2012.

[15] M. Shafie-khah and J. P. Catalão, "A stochastic multi-layer agent-based model to study electricity market participants behavior," IEEE Trans. Power Syst., vol. 30, no. 2, pp. 867-881, Mar. 2015.

[16] D. Menniti, A. Pinnarelli, and N. Sorrentino, "Simulation of producers behaviour in the electricity market by evolutionary games," Elect. Power Syst. Research, vol. 78, no. 3, pp. 475-483, Mar. 2008.

[17] K. Dehghanpour, M. H. Nehrir, J. W. Sheppard, and N. C. Kelly, "Agentbased modeling in electrical energy markets using dynamic bayesian networks," IEEE Trans. Power Syst., vol. 31, no. 6, pp. 4744-4754, Nov. 2016.

[18] S. Vázquez, P. Rodilla, and C. Batlle, "Residual demand models for strategic bidding in european power exchanges: revisiting the methodology in the presence of a large penetration of renewables," Electric power systems research, vol. 108, pp. 178-184, 2014.

[19] M. Zugno, J. M. Morales, P. Pinson, and H. Madsen, "Pool strategy of a price-maker wind power producer," IEEE Trans. Power Syst., vol. 28, pp. 3440-3450, Aug. 2013.

[20] L. Baringo and A. J. Conejo, "Strategic offering for a wind power producer," IEEE Trans. Power Syst., vol. 28, pp. 4645-4654, Nov. 2013.

[21] _ - "Offering strategy of wind-power producer: A multi-stage riskconstrained approach," IEEE Trans. Power Syst., vol. 31, pp. 1420-1429, Mar. 2016.

[22] H. Ding, P. Pinson, Z. Hu, J. Wang, and Y. Song, "Optimal offering and operating strategy for a large wind-storage system as a price maker," IEEE Trans. Power Syst., 2017.

[23] L. Mitridati and P. Pinson, "Optimal coupling of heat and electricity systems: A stochastic hierarchical approach," in 2016 Int. Conf. Probabilistic Methods Applied to Power Syst. (PMAPS). IEEE, 2016.

[24] J. M. Morales, A. J. Conejo, H. Madsen, P. Pinson, and M. Zugno, Integrating Renewables in Electricity Markets: Operational Problems. New York, NY, USA: Springer, 2014.

[25] J. G. Crespo, "The theory of bid function equilibria and its application to spot markets for electricity," Ph.D. dissertation, University of North Carolina at Chapel Hill, 2001.

[26] G. S. Crawford, J. Crespo, and H. Tauchen, "Bidding asymmetries in multi-unit auctions: implications of bid function equilibria in the british spot market for electricity," Int. J. Ind. Organization, vol. 25, no. 6, pp. 1233-1268, Dec. 2007.

[27] M. Pelagatti, "Supply function prediction in electricity auctions," in Complex Models and Computational Methods in Statistics. Springer, 2013, pp. 203-213.

[28] F. Silva, B. Teixeira, T. Pinto, G. Santos, Z. Vale, and I. Praça, "Generation of realistic scenarios for multi-agent simulation of electricity markets," Energy, vol. 116, pp. 128-139, Dec. 2016.

[29] C. Ruiz, A. J. Conejo, and D. J. Bertsimas, "Revealing rival marginal offer prices via inverse optimization," IEEE Trans. Power Syst., vol. 28, pp. 3056-3064, Aug. 2013.

[30] K. P. Murphy, "Dynamic Bayesian networks: representation, inference and learning," Ph.D. dissertation, UC Berkeley, 2002. 
[31] G. F. Cooper, "The computational complexity of probabilistic inference using bayesian belief networks," Artificial Intell., vol. 42, no. 2-3, pp. 393-405, Mar. 1990.

[32] W. R. Gilks, S. Richardson, and D. Spiegelhalter, Markov Chain Monte Carlo in practice. London: Chapman \& Hall/CRC, 1996.

[33] F. Liang, C. Liu, and R. Carroll, Advanced Markov Chain Monte Carlo methods: learning from past samples. Wiley, 2011, vol. 714.

[34] C. Andrieu, N. De Freitas, A. Doucet, and M. I. Jordan, "An introduction to MCMC for machine learning," Mach. Learning, vol. 50, no. 1, pp. 5-43, Jan. 2003.

[35] S. L. Scott, "Bayesian methods for hidden markov models: Recursive computing in the 21st century," Journal of the American Statistical Association, vol. 97, no. 457, pp. 337-351, 2002.

[36] J. S. Liu and R. Chen, "Sequential monte carlo methods for dynamic systems," J. Amer. Stat. Assoc., vol. 93, no. 443, pp. 1032-1044, Sept. 1998.

[37] A. Doucet, N. de Freitas, and N. Gordon, Sequential Monte Carlo methods in practice. Springer, 2001.

[38] M. G. Bruno, Sequential Monte Carlo methods for nonlinear discretetime filtering, ser. Synthesis Lectures Signal Process. Morgan \& Claypool Publishers, 2013.

[39] P. Del Moral, A. Doucet, and A. Jasra, "Sequential Monte Carlo samplers," J. Roy. Stat. Soc. B, vol. 68, no. 3, pp. 411-436, June 2006.

[40] A. Doucet, S. Godsill, and C. Andrieu, "On sequential Monte Carlo sampling methods for Bayesian filtering," Stat. and Comp., vol. 10, no. 3, pp. 197-208, July 2000 .

[41] B. Eraker, "MCMC analysis of diffusion models with application to finance," J. Bus. \& Econ. Stat., vol. 19, no. 2, pp. 177-191, Apr. 2001.

[42] F. Gustafsson, F. Gunnarsson, N. Bergman, U. Forssell, J. Jansson, R. Karlsson, and P.-J. Nordlund, "Particle filters for positioning, navigation, and tracking," IEEE Trans. Signal Process., vol. 50, pp. 425-437, Feb. 2002.

[43] H. Järvinen, M. Laine, A. Solonen, and H. Haario, "Ensemble prediction and parameter estimation system: the concept," Quart. J. Roy. Meteorological Soc., vol. 138, no. 663, pp. 281-288, Jan. 2012.

[44] M. Laine, A. Solonen, H. Haario, and H. Järvinen, "Ensemble prediction and parameter estimation system: the method," Quart. J. Roy. Meteorological Soc., vol. 138, no. 663, pp. 289-297, Jan. 2012.

[45] Z. Ghahramani, "An introduction to hidden Markov models and Bayesian networks," Int. J. Pattern Recognition and Artificial Intell., vol. 15, no. 01, pp. 9-42, Feb. 2001.

[46] Z. Ghahramani, M. I. Jordan, and P. Smyth, "Factorial hidden Markov models," Mach. Learning, vol. 29, no. 2-3, pp. 245-273, 1997.

[47] C. Andrieu, A. Doucet, and R. Holenstein, "Particle Markov Chain Monte Carlo methods," J. Roy. Stat. Soc. B, vol. 72, no. 3, pp. 269342, June 2010

[48] Nord Pool. (2017, June) Single hourly order - Nord Pool spot. [Online]. Available: http://www.nordpoolspot.com/TAS/Dayahead-market-Elspot/Order-types/Hourly-bid/

[49] A. G. Petoussis, X.-P. Zhang, S. G. Petoussis, and K. R. Godfrey, "Parameterization of linear supply functions in nonlinear AC electricity market equilibrium modelspart I: Literature review and equilibrium algorithm," IEEE Trans. Power Syst., vol. 28, no. 2, pp. 650-658, May 2013.

[50] W. K. Hastings, "Monte Carlo sampling methods using Markov Chains and their applications," Biometrika, vol. 57, no. 1, pp. 97-109, Apr. 1970.

[51] A. F. Smith and G. O. Roberts, "Bayesian computation via the Gibbs sampler and related Markov Chain Monte Carlo methods," J. Roy. Stat. Soc. B, vol. 55, no. 1, pp. 3-23, 1993.

[52] J. Carpenter, P. Clifford, and P. Fearnhead, "Improved particle filter for nonlinear problems," IEEE Proc.-Radar, Sonar Navigation, vol. 146, no. 1, pp. 2-7, Feb. 1999

[53] F. Lindsten, R. Douc, and E. Moulines, "Uniform ergodicity of the particle Gibbs sampler," Scandinavian J. Stat., vol. 42, no. 3, pp. 775797, Sept. 2015

[54] C. Ordoudis, P. Pinson, J. M. Morales, and M. Zugno, An Updated Version of the IEEE RTS 24-Bus System for Electricity Market and Power System Operation Studies. Technical University of Denmark, 2016.

[55] A. Belloni and V. Chernozhukov, "On the computational complexity of MCMC-based estimators in large samples," Ann. Stat., vol. 37, no. 4, pp. 2011-2055, Aug. 2009.

[56] E. Mossel and A. Sly, "Rapid mixing of Gibbs sampling on graphs that are sparse on average," in Proc. 19th Аппи. ACM-SIAM Symp. Discrete Algorithms. Society for Industrial and Applied Mathematics, 2008, pp. 238-247.
[57] C. M. De Sa, C. Zhang, K. Olukotun, and C. Ré, "Rapidly mixing Gibbs sampling for a class of factor graphs using hierarchy width," in Advances in Neural Information Processing Systems, 2015, pp. 3097-3105.

[58] M. K. Cowles and B. P. Carlin, "Markov chain Monte Carlo convergence diagnostics: a comparative review," J. Amer. Stat. Assoc., vol. 91, no. 434, pp. 883-904, June 1996.

[59] C. P. Robert, T. RydÉn, and D. M. Titterington, "Convergence controls for mcmc algorithms, with applications to hidden markov chains," $J$. Stat. Comput. Simulation, vol. 64, no. 4, pp. 327-355, 1999.

[60] G. Casella and C. P. Robert, "Rao-Blackwellisation of sampling schemes," Biometrika, vol. 83, no. 1, pp. 81-94, 1996.

[61] N. De Freitas, P. Højen-Sørensen, M. I. Jordan, and S. Russell, "Variational MCMC," in Proc. 17th Conf. Uncertainty in Artificial Intell. Morgan Kaufmann Publishers Inc., 2001, pp. 120-127.

[62] S. G. Walker, P. Damien, P. W. Laud, and A. F. Smith, "Bayesian nonparametric inference for random distributions and related functions," J. Roy. Stat. Soc. B, vol. 61, no. 3, pp. 485-527, 1999.

[63] R. Douc and O. Cappé, "Comparison of resampling schemes for particle filtering," in Proc. 4th Int. Symp. Image and Signal Process. and Anal. (ISPA). IEEE, 2005, pp. 64-69.

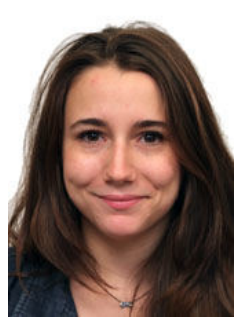

Lesia Mitridati received the Master degree in Science and Executive Engineering from Mines ParisTech, France, in 2015. She is currently pursuing the Ph.D. degree at the department of Electrical Engineering of the Technical University of Denmark (DTU). Her research interests include electricity markets, multi-energy systems, decision making under uncertainty and game theory.

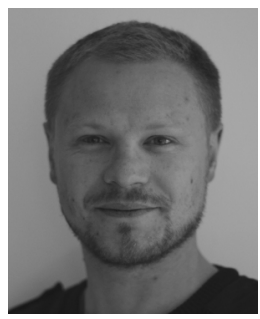

Pierre Pinson (M11-SM13) received the M.Sc. degree in applied mathematics from the National Institute for Applied Sciences (INSA Toulouse, France) and the Ph.D. degree in energetics from Ecole des Mines de Paris (France). He is a Professor at the Technical University of Denmark (DTU), Centre for Electric Power and Energy, Department of Electrical Engineering, also heading a group focusing on Energy Analytics \& Markets. His research interests include among others forecasting, uncertainty estimation, optimization under uncertainty, decision sciences, and renewable energies. He acts as an Editor for the International Journal of Forecasting, and for Wind Energy. 